\title{
On Dihedral Angles of a Simplex
}

\author{
H. Maehara ${ }^{1}$ \\ ${ }^{1}$ Ryukyu University, Nishihara, Okinawa, Japan \\ Correspondence: H. Maehara, Ryukyu University, Nishihara, Okinawa 903-0129, Japan. E-mail: \\ hmaehara@edu.u-ryukyu.ac.jp
}

Received: March 11, 2013 Accepted: April 24, 2013 Online Published: May 3, 2013

doi:10.5539/jmr.v5n2p79 URL: http://dx.doi.org/10.5539/jmr.v5n2p79

\begin{abstract}
For an $n$-simplex, let $\alpha, \beta$ denote the maximum, and the minimum dihedral angles of the simplex, respectively. It is proved that the inequality $\alpha \leq \arccos (1 / n) \leq \beta$ always holds, and either side equality implies that the $n$ simplex is a regular simplex. Similar inequalities are also given for a star-simplex, which is defined as a simplex that has a vertex (apex) such that the angles between distinct edges incident to the apex are all equal. Further, an explicit formula for the dihedral angle of a star-simplex between two distinct facets sharing the apex in common is presented in terms of the angle between two edges incident to the apex.
\end{abstract}

Keywords: dihedral angle, star-simplex, lateral angle

\section{Introduction}

Let $\sigma$ be an $n$-dimensional simplex ( $n$-simplex) in $\mathbb{R}^{n}, n \geq 2$, and let $f_{i}, f_{j}$ be two distinct facets of $\sigma$. The dihedral angle $\angle\left(f_{i}, f_{j}\right)$ between the facets $f_{i}, f_{j}$ is defined as the supplement of the angle between the unit outer normal vectors of the facets $f_{i}, f_{j}$. For $n \geq 3$, the sum of $\left(\begin{array}{c}n+1 \\ 2\end{array}\right)$ dihedral angles of an $n$-simplex is not constant. Indeed, the following holds (see Gaddum, 1952, 1956).

$\triangleright$ The sum of the $\left(\begin{array}{c}n+1 \\ 2\end{array}\right)$ dihedral angles of an $n$-simplex lies between $\left\lceil\frac{n^{2}-1}{4}\right\rceil \pi$ and $\left(\begin{array}{l}n \\ 2\end{array}\right) \pi$, and the sum can take any value in this range.

A dihedral angle is called acute (resp. nonobtuse) if the angle is less than (resp. not greater than) $\pi / 2$. The next result seems first appeared in Fielder (1954), and rediscovered again in Leng (2003).

$\triangleright$ Every $n$-simplex has at least $n$ acute dihedral angles.

For an $n$-simplex $\sigma$, let $\alpha=\alpha(\sigma), \beta=\beta(\sigma)$ denote the minimum value and the maximum value of the dihedral angles in $\sigma$, respectively. If $\sigma$ is a regular $n$-simplex, then $\alpha=\beta=\arccos \frac{1}{n}$. In the 2-dimensional case $n=2$, since the sum of the interior angles of a triangle is $\pi$, we have $\alpha \leq \pi / 3 \leq \beta$ and $\alpha=\pi / 3 \Leftrightarrow \beta=\pi / 3$. A similar assertion also holds in $n \geq 3$, though the sum of dihedral angles are not constant. The next is the main result of this paper.

Theorem 1 For every $n$-simplex, $\alpha \leq \arccos \frac{1}{n} \leq \beta$ holds. Moreover, if either side equality holds, then the other side equality also holds and the simplex becomes a regular simplex.

We present a similar result for a family of star-simplexes. A star-simplex with vertex angle $\theta$ is defined to be a simplex that has a vertex $v$ such that the plane angle between any two distinct edges incident to $v$ is equal to $\theta$. The vertex $v$ is called the apex of the star-simplex. If those edges incident to the apex are of the same length, then the star-simplex is called a regular star-simplex. In a star-simplex, the dihedral angles between two distinct facets sharing the apex in common are all equal, and their common value is called the lateral angle of the star-simplex.

Theorem 2 In an n-dimensional star-simplex with vertex angle $\theta$, the lateral angle $\delta=\delta(\theta)$ is given by

$$
\cos \delta=\frac{\cos \theta}{1+(n-2) \cos \theta} .
$$

It follows from (1) that $\delta(\theta)$ is a monotone increasing function of $\theta$ in $0 \leq \theta \leq \arccos \frac{-1}{n-1}$, and

$$
\delta(0)=\arccos \frac{1}{n-1}
$$




$$
\begin{gathered}
\delta\left(\frac{\pi}{3}\right)=\arccos \frac{1}{n} \\
\delta\left(\frac{\pi}{2}\right)=\pi / 2 \\
\delta\left(\arccos \frac{-1}{n-1}\right)=\pi .
\end{gathered}
$$

Theorem 3 An n-dimensional star-simplex with vertex angle $\theta$ (resp. lateral angle $\delta$ ) exists if and only if

$$
0<\theta<\arccos \frac{-1}{n-1}\left(\text { resp. } \arccos \frac{1}{n-1}<\delta<\pi\right) .
$$

Let $\varphi(\delta)=\arccos \left(\frac{1}{n} \sqrt{n-n(n-1) \cos \delta}\right)$. This is the other value of dihedral angles in a regular star-simplex with lateral angle $\delta$. Note that $\varphi(\delta)$ is strictly monotone decreasing in $\arccos \frac{1}{n-1}<\delta<\pi$, and $\varphi\left(\arccos \frac{1}{n}\right)=\arccos \frac{1}{n}$.

Theorem 4 For an $n$-dimensional star-simplex $\sigma$ with vertex angle $\theta$, the following holds:

(1) If $0<\theta<\pi / 3$ (i.e. $\arccos \frac{1}{n-1}<\delta<\arccos \frac{1}{n}$ ), then $0<\alpha \leq \delta<\varphi(\delta) \leq \beta<\pi-\delta$, and $\beta=\varphi(\delta)$ implies that $\sigma$ is a regular star-simplex.

(2) If $\pi / 3 \leq \theta<\pi / 2$ (i.e. $\arccos \frac{1}{n} \leq \delta<\pi / 2$ ), then $0<\alpha \leq \varphi(\delta) \leq \delta \leq \beta<\pi-\delta$, and $\alpha=\varphi(\delta)$ implies that $\sigma$ is a regular star-simplex.

(3) If $\pi / 2 \leq \theta<\arccos \frac{-1}{n-1}$ (i.e. $\pi / 2 \leq \delta<\pi$ ), then $0<\alpha \leq \varphi(\delta)<\delta=\beta$, and $\alpha=\varphi(\delta)$ implies that $\sigma$ is a regular star-simplex.

\section{Proof of Theorem 1}

Let $S(O, x) \subset \mathbb{R}^{n}$ denote a sphere with center $O$ and radius $x$.

Lemma 1 For an n-simplex $\sigma$, let $\overrightarrow{O P_{0}}, \overrightarrow{O P_{1}}, \ldots, \overrightarrow{O P_{n}}$ be the unit outer normal vectors of the facets of $\sigma$. Then, $P_{0}, \ldots, P_{n}$ span a simplex that contains $O$ in its interior.

Proof. We may suppose that $S(O, 1)$ is the inscribed sphere of $\sigma$, and the $n+1$ points $P_{0}, \ldots, P_{n}$ are the contact points of $S(O, 1)$ with the $n+1$ facets of $\sigma$. Then, no closed hemisphere of $S(O, 1)$ can contain these $n+1$ points, for otherwise, the inscribed sphere $S(O, 1)$ of $\sigma$ can slip out of the simplex $\sigma$. Hence, $O$ is an interior point of the simplex spanned by $P_{0}, \ldots, P_{n}$.

Let us recall here some values concerning a regular simplex. If a regular $n$-simplex has unit circumradius, then

$\circ$ the radius of its inscribed sphere is equal to $1 / n$,

$\circ$ its edge-length is equal to $l(n):=\sqrt{2(n+1) / n}$, and

$\circ$ its dihedral angle is equal to $\arccos \frac{1}{n}$.

Note that $l(n)$ is strictly monotone decreasing in $n$, and the edge-length of a regular $n$-simplex with circumradius $R$ is given by $R \cdot l(n)$.

For a set $V$ of $n+1$ points on a unit sphere $S(O, 1) \subset \mathbb{R}^{n}$, let $a(V), b(V)$ denote the minimum value and the maximum value of the Euclidean distance $|P Q|$ for $P, Q \in V, P \neq Q$. If $V$ spans a regular simplex, then we have $a(V)=b(V)=l(n)$.

Lemma 2 Suppose that a set $V$ of $n+1$ points on $S(O, 1) \subset \mathbb{R}^{n}$ spans an $n$-simplex $\langle V\rangle$ that contains $O$ in its interior. Then

(1) $a(V) \leq l(n)$, and if the equality holds, then $\langle V\rangle$ is a regular $n$-simplex.

(2) $b(V) \geq l(n)$, and if the equality holds, then $\langle V\rangle$ is a regular $n$-simplex.

Proof. (1) We use the following result in Deza and Maehara (1994):

(*) For any $m$ points $P_{1}, P_{2}, \ldots, P_{m}$ on $S(O, R)$, we have

$$
m^{2} R^{2} \geq \sum_{i<j}\left|P_{i} P_{j}\right|^{2},
$$

and the equality holds if and only if $\frac{1}{m} \sum P_{i}=O$. 
Applying (*) to the point set $V$ on $S(O, 1)$, we have $(n+1)^{2} \geq\left(\begin{array}{c}n+1 \\ 2\end{array}\right) a(V)^{2}$. This implies that $a(V) \leq l(n)$, and the equality holds only when $|P Q|=l(n)$ for all $P, Q \in V, P \neq Q$, which implies that $\langle V\rangle$ is a regular $n$-simplex.

(2) Proof is by induction on the dimension $n$. For $n=2$, (2) can be easily seen. Suppose that (2) is true for every $(n-1)$-simplex, and let us consider the $n$-dimensional case. We use the circumradius-inradius inequality for an $n$-simplex (see, e.g., Klamkin \& Tsintsifas, 1979):

(**) The radius $r$ of the inscribed sphere and the radius $R$ of the circumscribed sphere of an $n$-simplex always satisfy $r \leq R / n$.

Let $\tau=\langle V\rangle$. Since $O$ is an interior point of $\tau$, there is an $x_{0}>0$ such that $S\left(O, x_{0}\right)$ is tangent to a facet $f$ of $\tau$ and $S\left(O, x_{0}\right) \subset \tau$. This $x_{0}$ must be smaller than or equal to the radius $r_{0}$ of the inscribed sphere of $\tau$. Since $r_{0} \leq 1 / n$ by $(* *)$, we have $\sqrt{1-x_{0}^{2}} \geq \sqrt{1-r_{0}^{2}} \geq \sqrt{1-(1 / n)^{2}}$. The edge length of a regular $(n-1)$-simplex with circumradius $\sqrt{1-(1 / n)^{2}}$ is given by $\sqrt{1-(1 / n)^{2}} \cdot l(n-1)$, which is equal to $l(n)$ as easily verified. Note that the contact point of $S\left(O, x_{0}\right)$ and the facet $f$ is the circum-center of the facet $f$, and it is an interior point of $f$. Hence, we can apply the inductive hypothesis to the set $V_{f}$ of $n$ vertices of the facet $f$ on a sphere of radius $\sqrt{1-x_{0}^{2}}$ in $\mathbb{R}^{n-1}$. Therefore,

$$
b\left(V_{f}\right) \geq \sqrt{1-x_{0}^{2}} \cdot l(n-1) \geq \sqrt{1-(1 / n)^{2}} \cdot l(n-1)=l(n) .
$$

Thus, $b(V) \geq b\left(V_{f}\right) \geq l(n)$.

Now, suppose that $b(V)=l(n)$. Then, for $0<x<1 / n$, no facet of $\tau$ can touch $S(O, x)$, for otherwise, we have $b\left(V_{f}\right)>l(n)$ for some facet $f$ of $\tau$, as easily seen. Hence we can deduce, from $r \leq 1 / n$, that the sphere $S(O, 1 / n)$ must be tangent to all facets of $\tau$. In this case, $b(V)=l(n)$ implies that all edge-lengths of $\tau$ are equal to $l(n)$, and $\tau$ is a regular $n$-simplex.

Proof [Proof of Theorem 1]. Let $\sigma$ be an $n$-simplex and $f_{0}, f_{1}, \ldots, f_{n}$ be the facets of $\sigma$. Let $\overrightarrow{O P}_{i}$ be the unit outer normal vectors of the facets $f_{i}$. Then $P_{i} \in S(O, 1)$. Let $V=\left\{P_{0}, \ldots, P_{n}\right\}$, and $\tau$ be the simplex spanned by $V$. By Lemma $1, O$ is an interior point of $\tau$. The dihedral angle $\angle\left(f_{i}, f_{j}\right)$ of the facets $f_{i}, f_{j}(i \neq j)$ and the angle $\angle P_{i} O P_{j}$ are related as

$$
\angle\left(f_{i}, f_{j}\right)=\pi-\angle P_{i} O P_{j} .
$$

By the cosine law, $\angle P_{i} O P_{j}=\arccos \left(1-\frac{1}{2}\left|P_{i} P_{j}\right|^{2}\right)$, and $\left|P_{i} P_{j}\right|=l(n)$ if and only if $\angle P_{i} O P_{j}=\arccos \frac{-1}{n}$. Since $\arccos x$ is monotone decreasing for $0<x<\pi$, it follows from the inequality $a(V) \leq l(n) \leq b(V)$ in Lemma 2 that the minimum value of $\angle P_{i} O P_{j}$ is less than or equal to $\arccos \frac{-1}{n}$ and the maximum value of $\angle P_{i} O P_{j}$ is greater than or equal to $\arccos \frac{-1}{n}$. Now, since

$$
\arccos \frac{1}{n}=\pi-\arccos \frac{-1}{n}
$$

the theorem follows.

\section{Proof of Theorems 2 and 3}

Proof [Proof of Theorem 2]. Let $\left\langle v_{0}, v_{1}, \ldots, v_{n}\right\rangle$ be an $n$-dimensional star-simplex with vertex angle $\theta$, and suppose $v_{0}$ is the apex. Let $\boldsymbol{a}_{i}=\overrightarrow{v_{0} \vec{v}_{i}}, i=1,2, \ldots, n$. To compute the lateral angle $\delta$, we may suppose that $\left|v_{0} v_{i}\right|=1$ for $i=1,2, \ldots, n$. Let $\boldsymbol{n}_{i}$ denote the unit outer normal vector of the facet opposite to the vertex $v_{i}$. Then $\cos \delta=-\boldsymbol{n}_{1} \cdot \boldsymbol{n}_{2}$. We can write $\boldsymbol{n}_{1}$ as

$$
\boldsymbol{n}_{1}=x_{2} \boldsymbol{a}_{2}+x_{3} \boldsymbol{a}_{3}+\cdots+x_{n} \boldsymbol{a}_{n}+y \boldsymbol{a}_{1}
$$

with some $x_{2}, \ldots, x_{n}, y \in \mathbb{R}$. Since $\boldsymbol{a}_{i} \cdot \boldsymbol{n}_{1}=0$ for $i \neq 1$ and $\boldsymbol{a}_{i} \cdot \boldsymbol{a}_{j}=\cos \delta$ for $i \neq j$, we have

$$
\begin{aligned}
0 & =x_{i}+\left(x_{2}+x_{3} \cdots+x_{n}-x_{i}\right) \cos \delta+y \cos \delta \\
& =x_{i}(1-\cos \delta)+T \cos \delta+y \cos \delta,
\end{aligned}
$$

where $T=x_{2}+x_{3}+\cdots+x_{n}$. Therefore, $x_{2}=x_{3}=\cdots=x_{n}$. Thus, we may put $\boldsymbol{n}_{1}$, and (by symmetry) $\boldsymbol{n}_{2}$ as

$$
\begin{aligned}
& \boldsymbol{n}_{1}=x\left(\boldsymbol{a}_{2}+\boldsymbol{a}_{3}+\cdots+\boldsymbol{a}_{n}\right)+y \boldsymbol{a}_{1}, \\
& \boldsymbol{n}_{2}=x\left(\boldsymbol{a}_{1}+\boldsymbol{a}_{3}+\cdots+\boldsymbol{a}_{n}\right)+y \boldsymbol{a}_{2} .
\end{aligned}
$$


Since $\boldsymbol{a}_{2} \cdot \boldsymbol{n}_{1}=0$ and $\boldsymbol{n}_{1} \cdot \boldsymbol{n}_{1}=1$, we have

$$
\begin{aligned}
& 0=x(1+(n-2) \cos \theta)+y \cos \theta, \\
& 1=x^{2}(n-1+(n-1)(n-2) \cos \theta)+y^{2}+2 x y(n-1) \cos \theta .
\end{aligned}
$$

Subtracting $(2) \times(n-1) x$ from (3), we have

$$
1=y^{2}+x y(n-1) \cos \theta
$$

On the other hand,

$$
\boldsymbol{n}_{1} \cdot \boldsymbol{n}_{2}=y^{2} \cos \theta+2 x y(1+(n-2) \cos \theta)+x^{2}\left(n-2+\left(n^{2}-3 n+3\right) \cos \theta\right) .
$$

Using (3),

$$
\begin{aligned}
\boldsymbol{n}_{1} \cdot \boldsymbol{n}_{2}-1 & =y^{2}(\cos \theta-1)+x^{2}(-1+\cos \theta)+2 x y(1-\cos \theta) \\
& =-(x-y)^{2}(1-\cos \theta) .
\end{aligned}
$$

Hence

$$
\boldsymbol{n}_{1} \cdot \boldsymbol{n}_{2}=-(x-y)^{2}(1-\cos \theta)+1
$$

Let $t=(-\cos \theta) /(1+(n-2) \cos \theta)$. Then, $x=$ ty by (2), and substituting this in (4), we have

$$
y^{2}=(1+t(n-1) \cos \theta)^{-1} .
$$

Now, from (5) we have

$$
\boldsymbol{n}_{1} \cdot \boldsymbol{n}_{2}=-y^{2}(t-1)^{2}(1-\cos \theta)+1=\frac{-(t-1)^{2}(1-\cos \theta)}{1+t(n-1) \cos \theta}+1 .
$$

Simplifying this, we get

$$
\boldsymbol{n}_{1} \cdot \boldsymbol{n}_{2}=\frac{-\cos \theta}{1+(n-2) \cos \theta} .
$$

Since $\cos \delta=-\boldsymbol{n}_{1} \cdot \boldsymbol{n}_{2}$, we have the theorem.

Proof [Proof of Theorem 3]. It is enough to show that an $n$-dimensional star-simplex of lateral angle $\delta$ exists if and only if

$$
\arccos \frac{1}{n-1}<\delta<\pi
$$

The necessity of $\delta<\pi$ is obvious. Let $\sigma$ be an $n$-dimensional star-simplex with lateral angle $\delta$, and let $f_{0}, f_{1}, \ldots, f_{n}$ be the facets of $\sigma, f_{0}$ be the facet opposite to the apex. Let $\overrightarrow{O P}_{i}$ be the unit outer normal vectors of the facets $f_{i}$. Then $P_{i} \in S(O, 1)$, and the simplex $\left\langle P_{1}, \ldots, P_{n}\right\rangle$ is a regular $(n-1)$-simplex. By Lemma 1 , the simplex $\left\langle P_{0}, P_{1}, \ldots, P_{n}\right\rangle$ contains $O$ in its interior. Hence its facet $\left\langle P_{1}, \ldots, P_{n}\right\rangle$ does not contain $O$. Therefore, the circumradius of the facet $\left\langle P_{1}, \ldots, P_{n}\right\rangle$ is less than 1 . Let $Q$ be the circumcenter of $\left\langle P_{1}, \ldots, P_{n}\right\rangle$. Since this facet is a regular $(n-1)$-simplex, we have $\angle P_{1} Q P_{2}=\arccos \frac{-1}{n-1}$. Since $\angle P_{1} O P_{2}<\angle P_{1} Q P_{2}$, we have $\angle P_{1} O P_{2}<\arccos \frac{-1}{n-1}$. Hence

$$
\delta=\pi-\angle P_{1} O P_{2}>\pi-\arccos \frac{-1}{n-1}=\arccos \frac{1}{n-1} .
$$

Thus, $\delta>\arccos \frac{1}{n-1}$.

Proof of the converse is easy now, and it is omitted.

\section{Proof of Theorem 4}

Lemma 3 Let $\tau$ denote a (variable) $n$-dimensional star-simplex with lateral angle $\delta$, and let $\omega_{1}, \ldots, \omega_{n}$ be the dihedral angles between the facet opposite to the apex and other facets. Then (i) $\inf _{\tau} \min _{i} \omega_{i}=0, \sup _{\tau} \max _{i} \omega_{i}=\pi-\delta$, and (ii) $\max _{\tau} \min _{i} \omega_{i}=\min _{\tau} \max _{i} \omega_{i}=\varphi(\delta)$.

Proof. Let $f_{0}, f_{1}, \ldots, f_{n}$ be the facets of $\tau, f_{0}$ be the facet opposite to the apex, and let $\overrightarrow{O P}_{i}$ be the unit outer normal vector of $f_{i}$. Then $P_{i} \in S(O, 1)$. Let $P_{i}^{*} \in S(O, 1)$ denote the antipodal point of $P_{i}$. Since $\angle\left(f_{i}, f_{j}\right)=\pi-\angle P_{i} O P_{j}$, we have

$$
\angle\left(f_{i}, f_{j}\right)=\angle P_{i} O P_{j}^{*}
$$


Since $\angle\left(f_{i}, f_{j}\right)=\delta$ for $1 \leq i<j \leq n$, we may put

$$
\omega_{i}=\angle\left(f_{i}, f_{0}\right)=\angle P_{i} O P_{0}^{*} \text { for } i=1,2, \ldots, n .
$$

Since the simplex $\left\langle P_{0}, P_{1}, \ldots, P_{n}\right\rangle$ contains $O$ in its interior by Lemma $1, O P_{0}^{*}$ passes through an interior point of the facet $\left\langle P_{1}, \ldots, P_{n}\right\rangle$. Hence, $\omega_{i}<\angle P_{1} O P_{2}=\pi-\delta$ for $i=1,2, \ldots, n$. If $P_{0}^{*}$ approaches $P_{1}$, then $\omega_{1} \rightarrow 0$, and $\omega_{2} \rightarrow \angle P_{1} O P_{2}=\pi-\delta$. Hence we have (i). Since the facet $\left\langle P_{1}, \ldots, P_{n}\right\rangle$ is a regular $(n-1)$ simplex, the maximum value of $\min _{i} \omega_{i}=\min _{i} \angle P_{i} O P_{0}^{*}$ is attained when $O P_{0}^{*}$ passes through the center $Q:=\frac{1}{n}\left(P_{1}+P_{2}+\cdots+P_{n}\right)$ of the facet $\left\langle P_{1}, \ldots, P_{n}^{i}\right\rangle$. Therefore, $\cos \left(\max _{\tau} \min _{i} \omega_{i}\right)=|O Q|$. Similarly, we have $\cos \left(\min _{\tau} \max \omega_{i}\right)=|O Q|$. Let us compute $|P Q|^{2}$.

$$
\begin{aligned}
|O Q|^{2}=\overrightarrow{O Q} \cdot \overrightarrow{O Q} & =\frac{1}{n^{2}}\left(\overrightarrow{O P_{1}}+\cdots+\overrightarrow{O P_{n}}\right) \cdot\left(\overrightarrow{O P_{1}}+\cdots+\overrightarrow{O P_{n}}\right) \\
& =\frac{1}{n^{2}}\left(n+2 \sum_{1 \leq i<j \leq n} \overrightarrow{O P_{i}} \cdot \overrightarrow{O P_{j}}\right) \\
& =\frac{1}{n^{2}}(n+n(n-1) \cos (\pi-\delta)) \\
& =\frac{1}{n^{2}}(n-n(n-1) \cos \delta) .
\end{aligned}
$$

Thus $|O Q|=\frac{1}{n} \sqrt{n-n(n-1) \cos \delta}$, and (ii) follows.

Proof [Proof of Theorem 4]. Let $\tau$ be a (variable) $n$-dimensional star-simplex with lateral angle $\delta$, and let $f_{0}, f_{1}, \ldots, f_{n}$ be the facets of $\tau, f_{0}$ opposite to the apex. Let $\omega_{i}=\angle\left(f_{i}, f_{0}\right)$.

(1) Suppose $0<\theta<\pi / 3$ (i.e. $\arccos \frac{1}{n-1}<\delta<\arccos \frac{1}{n}$ ). In this case, $\varphi(\delta)>\delta$. By Lemma 3, we have $\inf _{\tau} \min _{i} \omega_{i}=0$, and

$$
\max _{\tau} \min \left\{\delta, \omega_{1}, \ldots, \omega_{n}\right\}=\min \left\{\delta, \max _{\tau} \min \omega_{i}\right\}=\min \{\delta, \varphi(\delta)\}=\delta
$$

Hence $0<\alpha \leq \delta$. Similarly, we have $\sup _{\tau} \max _{i} \omega_{i}=\pi-\delta$ and

$$
\min _{\tau} \max \left\{\delta, \omega_{1}, \ldots, \omega_{n}\right\}=\max _{\tau}\{\delta, \varphi(\delta)\}=\varphi(\delta) .
$$

Therefore, $\varphi(\delta) \leq \beta<\pi-\delta$. If $\beta=\varphi(\delta)$, then by the proof of Lemma 3, we see that $\sigma$ is a regular star-simplex.

(2) Suppose $\pi / 3 \leq \theta<\pi / 2$ (i.e. $\arccos \frac{1}{n} \leq \delta<\pi / 2$ ). In this case, $\varphi(\delta) \leq \delta$, and similarly to Case 1 , we have $0<\alpha \leq \varphi(\delta) ; \delta \leq \beta<\pi-\delta$, and $\alpha=\varphi(\delta)$ implies that $\sigma$ is a regular star-simplex.

(3) Suppose $\pi / 2 \leq \theta<\arccos \frac{-1}{n-1}$ (i.e. $\pi / 2 \leq \delta<\pi$ ). Since $\left(\begin{array}{l}n \\ 2\end{array}\right)$ dihedral angles of $\tau$ are equal to $\delta \geq \pi / 2$ and since every $n$-simplex has at least $n$ acute dihedral angles, the remaining $\left(\begin{array}{c}n+1 \\ 2\end{array}\right)-\left(\begin{array}{l}n \\ 2\end{array}\right)=n$ dihedral angles must be all acute. Hence $\beta=\delta$. Clearly $0<\alpha \leq \varphi(\delta)$, and $\alpha=\varphi(\delta)$ implies that $\sigma$ is a regular star-simplex.

\section{References}

Deza, M., \& Maehara, H. (1994). A few applications of negative-type inequalities. Graphs Combin., 10, $255-262$.

Fiedler, M. (1954). Geometry of the simplex in $E_{n}$. I. Casopis Pěst. Mat., 79, 297-320.

Gaddum, J. W. (1952). The sums of the dihedral and trihedral angles in a tetrahedron. Amer. Math. Monthly, 59, 370-371.

Gaddum, J. W. (1956). Distance sums on a sphere and angle sums in a simplex. Amer. Math. Monthly, 63, 91-96.

Klamkin, M. S., \& Tsintsifas, G. A. (1979). The circumradius-inradius inequality for a simplex. Math. Mag., 52, 20-22.

Leng, G. (2003). The minimum number of acute dihedral angles of a simplex. Proc. Amer. Math. Soc., 131, 3039-3042. http://doi.org/10.1090/S0002-9939-03-06880-1 